\title{
MANEJO DE LA INCARCERACIÓN DEL PENE Y ESCROTO POR OBJETO METÁLICO
}

\author{
N. ALONSO GRACIA, O. BIELSA GALI, O. ARANGO TORO, E. DE LEÓN MORALES, \\ D. CAÑIIS SÁNCHEZ, A. GELABERT-MAS
}

Servicio y Cátedra de Urología. Hospital del Mar. UAB. Barcelona.

Actas Urol Esp. 27 (8): 633-636, 2003

\section{RESUMEN}

MANEJO DE LA INCARCERACIÓN DEL PENE Y ESCROTO POR OBJETO METÁLICO

La incarceración del pene requiere una actuación urgente para evitar lesiones mayores. En este artículo presentamos dos casos de incarceración de pene y escroto ocurridas en nuestro centro. Aportamos una revisión de la literatura donde se recogen multitud de objetos, e intentamos simplificar las distintas técnicas para su resolución.

PALABRAS CLAVE: Incarceración del pene. Objeto metálico. Lesión del pene.

\section{ABSTRACT}

\section{PENILE AND SCROTUM INCARCERATION BY METALLIC OBJECT}

Penile incarceration requires urgent management to prevent chief wound. In this article we present two penile and scrotum incarceration cases happened in our center. We contribute to a literature revision where lots of objects are reported. We try to simplify the different resolution techniques.

$\mathrm{L}^{2}$ a incarceración o estrangulación del pene por bandas o anillos es una ocurrencia universal. Multitud de objetos han sido descritos en las distintas series (anillos de boda, bandas de goma, tuercas metálicas, cojinetes, dispositivos de autocircuncisión e incluso cabellos) ${ }^{1-3}$. En los adultos estos objetos son colocados deliberadamente para intensificar la respuesta sexual, como autotratamiento de la disfunción eréctil o por problemas psiquiátricos ${ }^{4}$. En los niños, estos utensilios son colocados en algunas ocasiones, intencionalmente por los padres para prevenir la enuresis y la incontinencia, pero en otras, no es más que un experimento infantil ${ }^{5}$. La extracción de estos objetos requiere ingenio para realizarlo de una manera simple, efectiva y con la menor repercusión para el paciente, pero también depende en algunos casos del material disponible. A continuación aportamos dos casos de incarceración de pene y escroto.

\section{CASOS CLÍNICOS}

Caso $n^{\circ} 1$. Un varón de 60 años, acudió al servicio de urgencias por imposibilidad de extracción de anillo colocado en raíz de pene y escroto hacía 48 horas. El paciente estaba sereno y no refería antecedentes psíquicos. A la exploración se apreció un anillo de acero de $1 \mathrm{~cm}$ de espesor y aproximadamente $4 \mathrm{~cm}$ de diámetro, sin cierre, que incluía pene y escroto y condicionaba un importante edema. No presentaba signos de necrosis ni lesiones cutáneas. Tampoco refería ninguna dificultad a la micción. Se intentaron maniobras de reducción del edema siendo impo- 
sible la extracción. Tras los numerosos intentos se decidió optar por cortar el anillo. Su rotura presentó numerosas dificultades dado el material de acero del que estaba formado y el diámetro del mismo. Los primeros intentos con cizalla y con sierra para el acero fueron infructuosos. Se decidió utilizar una sierra radial a la que se le colocó un adaptador para disminuir las revoluciones y obtener un mejor control de la misma. El paciente fue protegido con un manta para evitar el contacto con las chispas y entre la piel y el anillo se colocó un protector metálico. Para reducir el sobrecalentamiento del material se realizó irrigación continua con suero. No se realizó bajo ningún tipo de anestesia, ya que el paciente se mantuvo colaborador en todo momento. Conseguimos realizar 2 cortes en el anillo logrando la extracción en aproximadamente 20 minutos. Debido a la nacionalidad extranjera del paciente no pudimos realizar controles posteriores (Figs. 1 y 2).

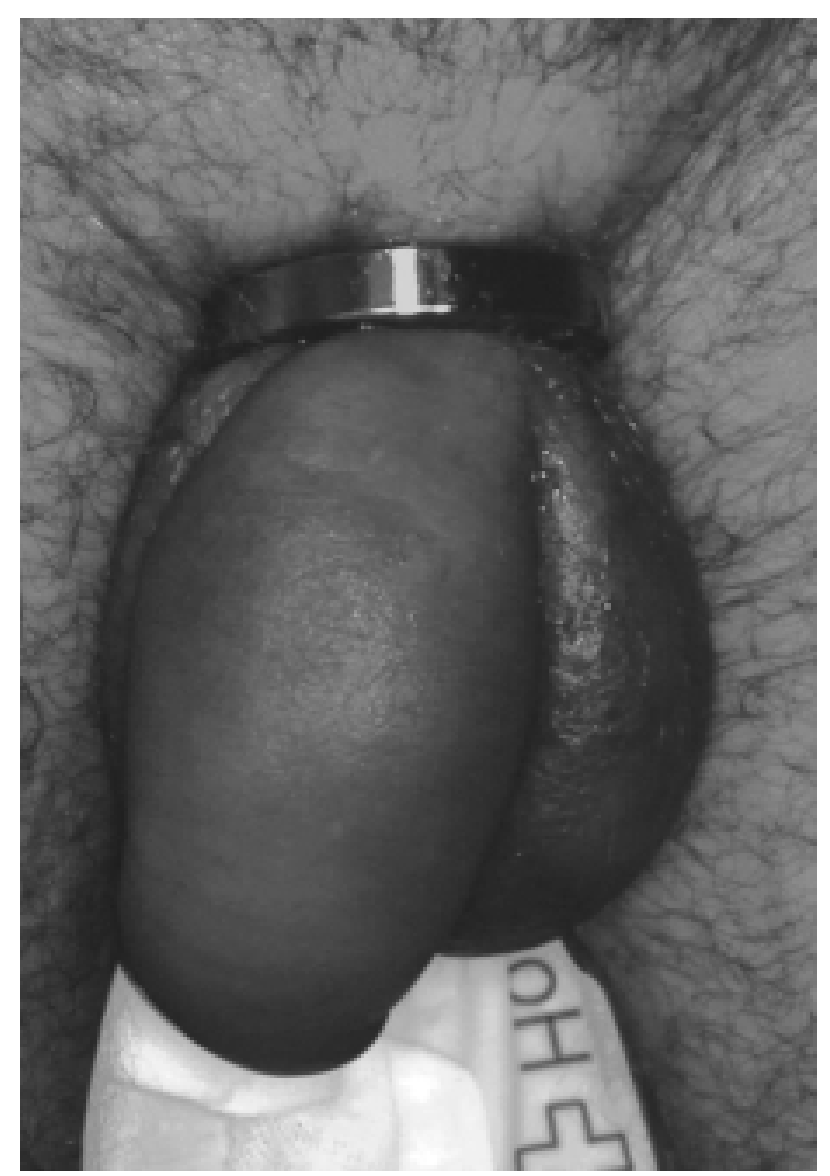

FIGURA 1. Pene y escroto incarcerados por anilla de acero.

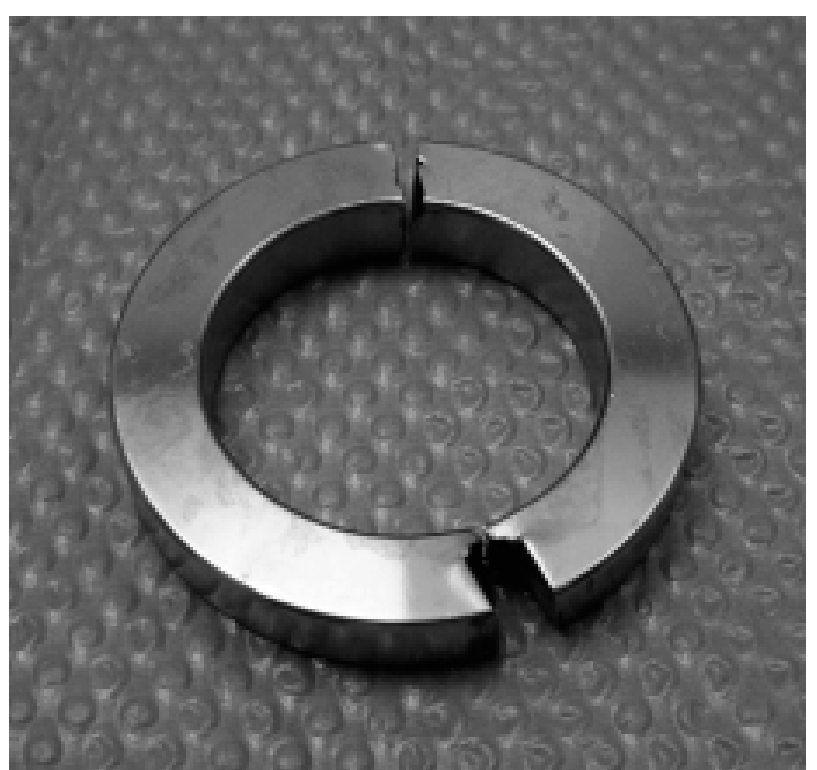

FIGURA 2. Anilla retirada de la base de pene y escroto.

Caso $n^{\circ}$ 2. Un varón de 55 años se presento en el hospital por dolor en el pene. El paciente refería la colocación de un anillo metálico hacía 18 horas. A la exploración presentaba edema distal sin signos de lesión cutánea. No presentaba problemas con la micción. Se intentaron maniobras para reducir el edema, sin conseguir la extracción. Dadas las características del anillo pudo ser serrado colocando previamente una protección entre el anillo y el pene. En pocas horas se consiguió la desaparición del edema. En los controles posteriores el paciente no presentó ninguna complicación preservando la función eréctil (Fig. 3).

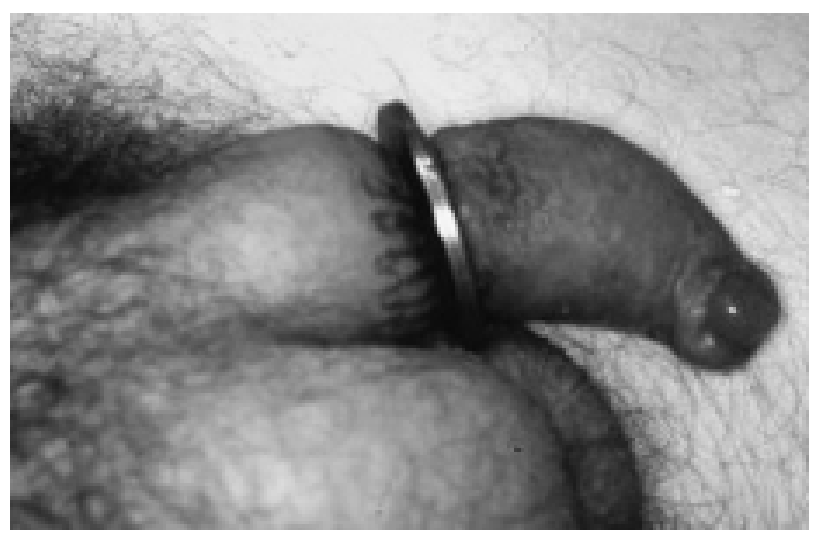

FIGURA 3. Anilla colocada en el cuerpo del pene. 


\section{DISCUSIÓN}

Los dispositivos colocados en el pene, y que condicionan la incarceración del mismo, es una circunstancia no muy frecuente que representa un desafío para el urólogo en el servicio de urgencias.

La mayoría de las veces, la colocación de un dispositivo en el pene flácido o semierecto, da como resultado la imposibilidad de retirarlo después de la erección. La fisiopatología de este proceso comienza con la dificultad del retorno venoso, que condiciona un edema distal y por tanto la imposibilidad de retirar el cuerpo extraño. Le sigue la afectación del aporte arterial de la piel y el tejido celular laxo. A continuación se lesiona el cuerpo esponjoso con la correspondiente lesión de la uretra. El cuerpo esponjoso es más vulnerable que los cuerpos cavernosos, dado que estos están protegidos por la aponeurosis de $\mathrm{Buck}^{6,7}$.

Bhat y cols. aportan la siguiente clasificación según el grado de las lesiones: ${ }^{1}$

- Grado 1. Edema distal del pene. No evidencia de úlcera cutánea ni lesión uretral.

- Grado 2. Lesión de piel, constricción del cuerpo esponjoso pero sin evidencia de lesión uretral. Edema distal con disminución de la sensibilidad del pene.

- Grado 3. Lesión de piel y uretra pero sin fístula uretral. Pérdida de la sensibilidad distal del pene.

- Grado 4. Completa división del cuerpo esponjoso condicionado una fístula uretral y constricción de los cuerpos cavernosos con perdida de la sensibilidad distal.

- Grado 5. Gangrena, necrosis o amputación de la porción distal del pene.

Nuestro paciente correspondería al grado 1 .

La evaluación de la incarceración del pene incluye la valoración de la temperatura, el color, la sensibilidad y el pulso distal. Para valorar el pulso es recomendable utilizar el Doppler. Otras exploraciones como la arteriografía son cuestionables por que además de tener poco valor retrasarían la extracción del cuerpo extraño ${ }^{8}$. Es recomendable realizar análisis de orina y cultivo para descartar enfermedades de transmisión sexual. En los casos severos cuando hay una lesión uretral evidente se debe colocar sonda suprapúbica y la uretra debe ser evaluada tan pronto como sea posible tras la retirada del anillo ${ }^{3-5,9}$. Es importante la evaluación del tejido desvitalizado. En caso de no evaluarlo puede dar lugar a infección y fístulas. Mc Graw y cols. en 1977 hacen referencia a la utilización de la lámpara de Wood con este fin. Tras la administración de fluoresceína intravenosa, la luz nos permite identificar los tejidos desvitalizados pudiendo llevar a cabo su exéresis ${ }^{14}$. Nosotros no hemos encontrado en los casos revisados ningún autor que haga referencia a la aplicación de esta técnica.

Podemos diferenciar básicamente dos grupos de cuerpos extraños: los no-metálicos y los metálicos. Los objetos no-metálicos son más fáciles de extraer pero sus lesiones son más severas ${ }^{2}$. Bhat y cols. observaron en su serie que el grupo de objetos metálicos presentaban lesiones grado $1 \mathrm{y}$ 2, y que el grupo de los no-metálicos estaban incluidos en los grados 3 y 4, dado que estos objetos son más finos y cortantes. Bhat también aprecia la diferenciación por edades, entre 5 y 16 años eran atendidos por objetos no metálicos y los pacientes con edades entre 15 y 56 años las lesiones eran producidas por objetos metálicos ${ }^{1}$.

Ante esta situación el especialista debe agudizar su ingenio y aprovechar los recursos disponibles. Detweiler dividió las técnicas de tratamiento en cuatro grupos: la técnica del cordón y sus variantes, técnicas de aspiración, corte del dispositivo y técnicas quirúrgicas ${ }^{4}$. La técnica del cordón fue inicialmente descrita por Flatt para retirar anillos del dedo traumático, posteriormente fue Bucy quien en 1968 aplicó la técnica para el pene incarcerado ${ }^{4}$. Desde entonces son varios los autores que han publicado el éxito de su aplicación ${ }^{5,7}$. Existen variantes como "la técnica de la cubierta" en la que emplean la banda de goma con la que se fijan las bolsas de diuresis de pierna, resultando menos traumático ${ }^{10}$. Esta técnica se empleó en aquellos pacientes cuyo tiempo de evolución era inferior a las $72 \mathrm{~h}$. La técnica de aspiración se puede emplear sola o combinada con la técnica del cordón. Se puede aplicar únicamente a tejido celular subcutáneo vaciando $\operatorname{linfa}^{7,11}$. En casos que lo precisen se puede aspirar el glande. El periodo de evolución es el más corto de los cuatro grupos. Cortar el dispositivo requiere tener a disposición material necesario, así como personal que sepa manejarlo. Distintos 
autores han publicado diferentes métodos: taladros, sierras, martillos, cinceles, etc. ${ }^{1-3,6,8,9}$. Como precaución hay que tener en cuenta la colocación de una protección entre la anilla y el pene y si la técnica lo precisa irrigación continua con suero para evitar el sobrecalentamiento. La cirugía queda reservada para el grado 5 . En casos extremos se ha descrito el "desguantado" del pene hasta la fascia de Buck, seguido de injertos cutáneos. También esta descrita la amputación y reimplante del pene con microcirugía ${ }^{4}$.

Tras la extracción del cuerpo extraño los cuidados postoperatorios deben ir encaminados a prevenir las infecciones y cuidar el tejido viable. Los antibióticos no están indicados si la epidermis y la uretra están intactas ${ }^{4}$.

La heparinización debe tenerse en cuenta si el pulso en las arterias del pene no aparece 30 minutos después de la retirada del cuerpo extraño ${ }^{1}$.

Las complicaciones tardías pueden ser fístulas, estenosis de uretra, y priapismo. La disminución de la sensibilidad normalmente se recupera en un plazo corto de tiempo.

Como conclusión podemos decir que la incarceración del pene es una lesión importante, sin embargo un correcto manejo de la misma puede conseguir un recuperación sin secuelas.

\section{REFERENCIAS}

1. BHAT AL, KUMAR A, MATHUR SC, GANGWAL KC.: Penile strangulation. Br J Urol 1991; 68: 618-621.

2. KIM SJ, CHUNG H, AHN HS, CHUNG DY, KIM YS.: Strangulation of the penis by a self-circumcision device. Urol Int 2002; 68: 197-198.

3. MHIRI MN, CHAKROUN Z.: Urethral injury secondary to penile strangulation by hair. Br J Urol 1992; 69: 319-320.
4. DETWEILER MB.: Penile incarceration with metal objects -a review of procedure choice based on penile trauma grade. Scand J Urol Nephrol 2001; 35: 212217.

5. VÄHÄSARJA VJ, HELLSTRÖM PA, SERLO W, KONTTURI MJ.: Treatment of penile incarceration by the string method: 2 case reports. J Urol 1993; 149: $372-373$.

6. MARTÍN A, REGADERA FJ, MARTÍN JL, RIVAS M, SAHAGUN JL, MARTINEZ FJ.: Incarceración de pene por objeto metálico en su base. Arch Esp de Urol 1994; 47: 730-732.

7. ALONSO D, RODRÍGUEZ LA, AMO JA, SANTOS J, DE CASTRO CJ, CAMACHO JJ et al.: Incarceración peneana por anillos metálicos. Actas Urol Esp 2000; 24: 262-264.

8. SNOY FJ, WAGNER SA, WOODSIDE JR, ORGEL MG, BORDEN TA.: Management of penile incarceration. Urology 1984; 24: 18-20.

9. PERABO FGE, STEINER G, ALBERS P, MÜLLER SC.: Treatment of penile strangulation caused by constricting devices. Urology 2002; 59: 137xiii$137 x v$.

10. DETWEILER MB, PERKINS D.: Penile incarceration: a wrapping technique. J Emerg Med 2001; 20: 159-161.

11. TUNDIDOR AM.: Incarceración del pene por objeto metálico pesado. Arch Esp de Urol 1998; 51: 483484.

12. SINHA BB.: Penile incarceration by a metallic object. Br J Surg 1988; 75: 33.

13. MOOREVILLE M, MELLER M.: Penile incarceration with barbell retaining ring. $J$ Urol 2001; 166: 618 .

14. McCRAW JB, MYERS B, SHANKLIN KD.: The value of fuorescein in predicting the viability of arterialized flaps. Plast Reconstr Surg 1977; 60: 710-719.

15. MOOREVILLE M, MELLER M. Re: Penile incarceration with barbell retaining ring. J Urol 2002; 167: 2531 .

Dra. N. Alonso Gracia

Servicio de Urología. Hospital del Mar

Po Marítimo, 25-29 - 08003 Barcelona

(Trabajo recibido el 25 octubre de 2002) 